\title{
PSICOTRÓPICOS: ERROS DE PRESCRIÇÃO E DISPENSAÇÃO EM FARMÁCIA DE UNIDADE BÁSICA DE SAÚDE PSICOTRÓPICOS: ERROS DE PRESCRIÇÃO E DISPENSAÇÃO
}

\author{
PSYCHOTROPICS: PRESCRIPTION AND DISPENSATION ERRORS IN A \\ PHARMACY OF BASIC HEALTH UNIT \\ PSYCHOTROPICS: PRESCRIPTION AND DISPENSATION ERRORS
}

Gisele da Silveira Lemos, Cleriane Santos Macêdo, Danilo Bomfim Miranda, Vyvian Dias Miranda Soares, Bianca Oliveira Souza

Universidade Estadual do Sudoeste da Bahia - UESB

\section{Abstract}

A medical prescription is a standardized legal document, which is extremely important in communication between prescribers and dispensers. The incompleteness of the information present in the prescription and dispensing leads to medication errors and risks to the health of patients in drug therapy.. The aim of this study was to assess compliance with the technical standards imposed by Ordinance No. 344/98 for the prescription and dispensing of psychotropic drugs in notifications of B1 prescriptions dispensed in a Basic Health Unit in the city of Jequié, Bahia. A cross-sectional study was carried out with document analysis, evaluating 249 notifications of B1 receipts, from August to October 2019. Data were tabulated in Excel and their analyzes performed in Statistical Package for the Social Science (SPSS). Notifications were absent in $96.0 \%$ of the patient's address, $99.2 \%$ of the amount of the drug was not written in full, and $100 \%$ of the signature of the person responsible for dispensing was absent. On the other hand, all standardization data for printing in notifications were found. The results show the negligence of the professionals involved regarding their role in complying with the law, leaving room for failures in the pharmacotherapeutic treatment.

Keywords: Psychotropic Drugs; Medication erros; Heatlh Centers.
Resumo

A prescrição médica é um documento padronizado de ordem legal, importante na comunicação entre prescritores e dispensadores. A incompletude de informações na prescrição $e$ dispensação leva aos erros de medicação e risco na terapia medicamentosa. O objetivo desse estudo foi avaliar o cumprimento das normas técnicas impostas pela Portaria número 344/98 para prescrição e dispensação de psicotrópicos nas notificações de receitas $B 1$ dispensadas em uma Unidade Básica de Saúde do município de Jequié, Bahia. Realizou-se um estudo transversal com análise documental, avaliando 249 notificações de receitas $B 1$, do período de agosto a outubro de 2019. Os dados foram tabulados no programa Excel e suas análises realizadas no Statistical Package for the Social Science (SPSS). As notificações apresentaram ausência em 96,0\% no endereço do paciente, 99,2 \% da quantidade do medicamento não estava escrita por extenso $e$ em 100\% estavam ausentes a assinatura do responsável pela dispensação. Em contrapartida, foram encontrados todos os dados de padronização para impressão nas notificações. Os resultados mostram a negligência dos profissionais envolvidos, quanto ao seu papel no cumprimento da lei, abrindo margem para falhas no tratamento farmacoterapêutico.

Palavras-chave: Psicotrópicos; Erros de medicação; Centros de Saúde. 
De acordo com a Organização Mundial de Saúde (OMS), os Psicotrópicos são substâncias que agem no Sistema Nervoso Central, e desta forma são capazes de produzir alterações de conduta, humor e cognição ${ }^{1}$. É fato que hoje se verifica um consumo crescente de medicamentos psicotrópicos decorrente de uma medicalização da sociedade, influenciada pelo surgimento de novos fármacos, prescrição excessiva por parte dos médicos o que pode gerar erros de medicação e consequentes eventos adversos (danos) aos pacientes ${ }^{2}$.

Os fármacos psicotrópicos são utilizados para tratamento de transtornos mentais, classificados pela OMS como: distúrbios com alta carga de morbidade, como depressão, distúrbios bipolares, esquizofrenia, transtornos de ansiedade, demência, transtornos por abuso de substâncias, deficiências intelectuais e os distúrbios comportamentais e de desenvolvimento que geralmente começam na infância e adolescência, incluindo o autismo ${ }^{3,4}$.

No 'Plano de Ação para a Saúde Mental 2013-2020', a OMS ${ }^{5}$ cita que uma em cada dez pessoas no mundo sofre de algum transtorno de saúde mental e que essas atingem aproximadamente 700 milhões de pessoas e representam $13 \%$ do total das doenças do mundo e que cerca de 90 milhões terão algum distúrbio pelo abuso ou dependência de psicotrópicos, no período 2013-2020.

Apesar dos medicamentos Psicotrópicos serem eficazes e seguros quando utilizados para fins terapêuticos em transtornos psíquicos e emocionais, o seu uso indiscriminado pode acarretar dependência Física e/ou Psíquica, reações adversas e eventos adversos ${ }^{6}$.

Neste sentido, torna-se necessário um controle rígido referente à aquisição e dispensação dos Psicotrópicos. Para isso existem documentos técnicos que regulamentam e descrevem o controle sanitário referente à fabricação, comercialização, prescrição e dispensação de substâncias e medicamentos sujeitos a controle especial, como a Portaria 344 de 12 de maio de 1998 da Secretaria de Vigilância Sanitária do Ministério da Saúde (SVS/MS) ${ }^{7,8}$.

A prescrição médica é uma ordem legal disponibilizada por profissionais habilitados e é dirigido ao profissional farmacêutico o qual a dispensa, ambos profissionais se tornam responsáveis pelos resultados produzidos por tais informações ${ }^{9}$. A farmácia é legalmente reconhecida como um estabelecimento de saúde e integra a rede de atenção primária à saúde ${ }^{10}$.
Assim, durante o processo de dispensação o profissional farmacêutico deve orientar o paciente quanto às dosagens corretas a serem administradas, as possíveis interações medicamentosas, as condições de armazenamento, possíveis reações adversas, além de analisar os aspectos legais e sanitários da prescrição assegurando o uso racional de medicamentos e prevenindo erros de medicação ${ }^{4,11}$.

Em contrapartida, diversos estudos têm demonstrado a incompletude e erros referentes às informações contidas em notificações de receitas de controle especial dispensadas em instituições públicas e privadas ${ }^{7,12,13,14,15,16,17}$. Tal fato pode ocasionar impactos negativos no que diz respeito ao uso racional desses medicamentos, pois podem contribuir para erros de medicação, abuso e uso ilícito ${ }^{18}$.

Dessa forma, considerando as consequências geradas pela incompletude e erros em notificações de receitas, este estudo teve como objetivo avaliar o cumprimento das normas técnicas impostas pela legislação vigente para a prescrição e dispensação dos psicotrópicos das notificações de receitas B1 dispensadas na farmácia comunitária de uma unidade básica de saúde do município de Jequié, Bahia.

\section{Metodologia}

Tipo de estudo e local da pesquisa

Estudo transversal, realizado em uma farmácia de Unidade Básica de Saúde localizada no município de Jequié, da região Sudoeste da Bahia. A farmácia funciona no horário de 07 às 17 horas de segunda a sexta-feira, com a presença de um profissional farmacêutico.

\section{Amostra e coleta de dados}

A coleta de dados foi realizada baseada na análise documental de notificações de receita B para uso de medicamentos psicotrópicos, disponibilizadas pela farmácia da Unidade Básica de Saúde. Após a dispensação do medicamento, a notificação de receita passa a ser documento do estabelecimento farmacêutico, para fins de escrituração semanal que deverá ser realizada pelo farmacêutico no Livro de Registro Específico e, para efeito de fiscalização e controle pela Autoridade Sanitária Municipal. O instrumento de coleta utilizado foi um formulário de avaliação estruturado, validado em estudo piloto, e 
utilizado em outros trabalhos ${ }^{15,16}$. A coleta de dados ocorreu durante o período de agosto a outubro de 2019, com prescrições dispensadas neste mesmo intervalo.

Variáveis

As variáveis ou grupos de variáveis estudadas foram:

- Itens obrigatórios da notificação de receita: cor da notificação (azul), sigla da unidade federativa, identificação numérica expedida pela autoridade sanitária competente local;

- Identificação do emitente: especialidade médica, assinatura do prescritor, nome com sua inscrição no Conselho Regional com a sigla da respectiva Unidade da Federação; ou nome da instituição, endereço completo e telefone;

- Informações do paciente: nome e endereço completo.

- Informações referente ao medicamento: nome, concentração, forma farmacêutica, via de administração, dose, frequência diária de administração, duração do tratamento, data da prescrição e instruções complementares sobre o modo de usar o medicamento;

- Itens de identificação do comprador: nome completo, número do documento de identificação, órgão emissor, endereço completo e telefone;

- Itens de preenchimento obrigatório pelo profissional dispensador: carimbo e assinatura do farmacêutico, data da dispensação e quantidade aviada;

- Identificação da gráfica: nome, endereço e C.N.P.J./ C.G.C. impressos no rodapé de cada folha do talonário, numeração inicial e final concedidas ao profissional ou instituição e o número da Autorização para confecção de talonários emitida pela Vigilância Sanitária local.
Análise de dados

Foi considerado "Sim", para o preenchimento completo do item analisado e "Não" para ausência da informação. O processamento dos dados foi realizado utilizando os programas Microsoft Office Excel ${ }^{\circledR}$, como banco de dados para transcrever as informações coletadas das notificações de receita e, para análise dos dados foi utilizado o programa SPSS ${ }^{\circledR}$ (Stastistical Package for the Social Sciences), versão 21.0, (IBM SPSS 20.0, 2011, Armonk, NY: IBM Corp.). A análise descritiva foi realizada estimando-se as frequências absolutas e relativas das variáveis categóricas.

\section{Resultados}

Foram avaliadas 249 notificações de receita de medicamentos psicotrópicos, presentes na lista B1, em farmácia de uma Unidade Básica de Saúde.

Informações obrigatórias da notificação de receita

Com relação a padronização da notificação de receita pela portaria $344 / 98^{6}$, alguns itens são obrigatórios, e estão destacados na tabela 1, estando todos impressos na forma adequada. O nome, endereço, o Cadastro Nacional de Pessoa Jurídica (CNPJ)/ e o Cadastro Geral de Contribuintes (CGC) devem estar impressos no rodapé do talonário, bem como, a numeração inicial e final conferida a instituição e/ou ao profissional prescritor para confecção dos talonários fornecido pela Vigilância Sanitária local. Além disso, os medicamentos psicotrópicos precisam ser prescritos em notificação de receita "B", de cor azul, para sua dispensação ${ }^{6}$.

Tabela 1 - Variáveis referentes à padronização de notificações de receita B dos medicamentos psicotrópicos, dispensados em uma farmácia de Unidade Básica de Saúde, agosto a outubro de 2019. Bahia, Brasil.

\begin{tabular}{lcc}
\hline Itens avaliados & Sim N (\%) & Não N (\%) \\
\hline Unidade federativa & $249(100)$ & $0(0)$ \\
Identificação numérica & $249(100)$ & $0(0)$ \\
Cor & $249(100)$ & $0(0)$ \\
Assinatura e carimbo do prescritor & $249(100)$ & $0(0)$ \\
Nome da gráfica & $249(100)$ & $0(0)$ \\
Endereço da gráfica & $249(100)$ & $0(0)$ \\
CNPJ e CGC da gráfica & $249(100)$ & $0(0)$
\end{tabular}


Numeração inicial e final

$\mathrm{N}^{\circ}$ da Autorização para Confecção de Talonários

Fonte: Elaboração dos autores (2020). CNPJ: Cadastro Nacional da Pessoa Jurídica. CGC: Cadastro Geral de Contribuintes. $\mathrm{N}^{\circ}$ : número

Itens de preenchimento obrigatório pelo médico prescritor

Dos itens de preenchimento obrigatório pelo médico prescritor verificou-se que das 249 notificações de receita $B$ avaliadas o item referente a data de expedição apresentou resultado insatisfatório com altas porcentagens de incompletude, tabela 2 . No que se refere as informações do paciente, o endereço do usuário foi o item mais ausente nas notificações. Em uma $(0,4 \%)$ das prescrições não foi possível a interpretação e identificação do paciente. Das notificações nas quais constava a especialidade médica, 66 (62,8\%) foram prescritas por psiquiatras.

Tabela 2 - Variáveis referentes aos dados do paciente e do emitente, de notificações de receita B1 dos medicamentos psicotrópicos, dispensados em farmácia de Unidade Básica de Saúde, agosto a outubro de 2019. Bahia. Brasil.

\begin{tabular}{lcc}
\hline Itens avaliados & Sim N (\%) & Não N (\%) \\
\hline Nome do usuário & $248(99,6)$ & $1(0,4)$ \\
Endereço usuário & $10(4,0)$ & $239(96,0)$ \\
Data de expedição & $83(33,3)$ & $156(62,7)$ \\
Identificação do emitente médico (n=156) & & \\
$\quad$ Nome do prescritor & $156(100)$ & $0(0)$ \\
$\quad$ CRM/RMS & $156(100)$ & $0(0)$ \\
$\quad$ Unidade federativa & $114(73,1)$ & $42(26,9)$ \\
Especialidade médica & $105(67,3)$ & $51(32,7)$ \\
Identificação do emitente IS (n=93) & & \\
$\quad$ Nome da instituição de saúde & $93(100)$ & $0(0)$ \\
Telefone da instituição de saúde & $87(93,5)$ & $6(6,5)$ \\
Endereço da instituição de saúde & $89(95,7)$ & $4(4,3)$ \\
\hline
\end{tabular}

Fonte: Elaboração dos autores (2020). CRM: Conselho Regional de Medicina; RMS: Registro Único do Ministério da Saúde.

Na Tabela 3 constam os itens referentes ao preenchimento do campo relacionado ao tratamento do paciente. Em 27 notificações de receita $(10,8 \%)$ ocorreu o preenchimento da substância junto com a dose por unidade posológica. Em todas as notificações que apresentavam forma farmacêutica o tratamento era prescrito em comprimidos, mas em $3(1,2 \%)$ essa informação foi preenchida apenas no campo da posologia. No que se refere a quantidade, o preenchimento correto foi observado em 137 (55,0\%) das notificações de receita, e das 112 
(45\%) que estavam ausentes foram observados problemas como substituição da quantidade e forma farmacêutica pelo número de caixas $(93,75 \%)$. Além disso, ainda relacionado a quantidade, observou-se que em 247 (99,2\%) notificações essa informação não estava escrita por extenso e 134 (53,8\%) estava preenchido em algarismos arábicos.

Tabela 3 - Variáveis referentes às informações do medicamento, de notificações de receita B dos medicamentos psicotrópicos, dispensados em farmácia de Unidade Básica de Saúde, agosto a outubro de 2019. Bahia, Brasil

\begin{tabular}{lcc}
\hline Itens avaliados & Sim N (\%) & Não N (\%) \\
\hline Nomenclatura oficial (DCB/DCI) & $230(92,4)$ & $19(7,6)$ \\
Forma farmacêutica & $88(35,3)$ & $161(64,7)$ \\
Quantidade & $137(55,0)$ & $112(45,0)$ \\
Quantidade em algarismos arábicos & $134(53,8)$ & $115(46,2)$ \\
Quantidade por extenso & $2(0,8)$ & $247(99,2)$ \\
Dose por unidade posológica & $249(100)$ & $0(0)$ \\
Posologia & $249(100)$ & $0(0)$ \\
\hline
\end{tabular}

Fonte: Elaboração dos autores (2020). DCB/DCl: Denominação Comum Brasileira/Denominação Comum Internacional.

Itens de preenchimento obrigatório pelo profissional dispensador

$\mathrm{Na}$ Tabela 4, estão descritos os dados referentes à dispensação do medicamento. Em $13(5,2 \%)$ das notificações com o preenchimento do telefone havia informação de que o paciente não possuía número para contato. Além disso, em todas as notificações não havia assinatura do profissional farmacêutico, nome e endereço do estabelecimento que realizou a dispensação.

Tabela 4 - Variáveis referentes aos dados da dispensação dos medicamentos de notificações de receita B1 dos medicamentos psicotrópicos, dispensados em farmácia de Unidade Básica de Saúde, agosto a outubro de 2019. Bahia, Brasil

\begin{tabular}{lcc}
\hline Itens avaliados & Sim N (\%) & Não N (\%) \\
\hline Nome do comprador & $247(99,2)$ & $2(0,8)$ \\
Número de identidade & $215(86,3)$ & $34(13,7)$ \\
Órgão emissor & $97(39,0)$ & $152(61,0)$ \\
Endereço do comprador & $211(84,7)$ & $38(15,3)$ \\
Telefone do comprador & $198(79,5)$ & $51(20,5)$ \\
Nome do estabelecimento farmacêutico & $0(0)$ & $249(100)$ \\
Endereço do estabelecimento farmacêutico & $0(0)$ & $249(100)$ \\
Assinatura do Responsável pela Dispensação & $0(0)$ & $249(100)$
\end{tabular}


Continuação...

Data do atendimento $\quad 249(100) \quad 0(0)$

Quantidade aviada $\quad 249(100) \quad 0(0)$

Fonte: Elaboração dos autores (2020).

\section{Discussão}

A presente pesquisa realizada em uma Unidade Básica de Saúde identificou a incompletude e erros de prescrição em notificações de receita $B$ de medicamentos psicotrópicos. Esse resultado deve ser observado com precaução por gestores e profissionais de saúde, uma vez que destaca a importância de reflexão sobre as práticas de segurança e uso racional de medicamentos que têm sido adotadas neste ambiente. Por outro lado, essas evidências apontam também para a importância de se fomentar a sensibilização dos profissionais a aderirem a uma cultura de segurança e reduzirem a ocorrência de erros de medicação ${ }^{19}$.

Com relação aos dados de padronização para impressão das notificações de receitas $B$, todas estavam corretas. A presença desses itens demonstra a autenticidade dos documentos, assegurando legalidade e legitimidade às notificações, desta forma, evita-se falsificações e fraudes como, a dispensação errônea desses medicamentos. De acordo com a Portaria $\mathrm{n}^{\circ}$ $344 / 98^{6}$, a identificação da gráfica, a Unidade Federativa, cor e identificação numérica, devem estar apropriadamente impressos nas notificações. Os resultados aqui detectados, estão em concordância com os encontrados por Mota e colaboradores $(2016)^{14}$, nos quais esses itens analisados estavam em sua totalidade preenchidos.

As informações necessárias para a terapia do paciente estão dispostas na prescrição e o prescritor é o responsável por estabelecê-las de maneira racional e compreensível ${ }^{20}$. A notificação de receita deve ser prescrita de forma legível, sem rasuras, e deve conter itens de preenchimento obrigatório pelo médico prescritor, como a identificação do paciente: nome completo, endereço e data de expedição, essas informações vão possibilitar um tratamento personalizado do paciente. Verificouse a inexistência de dados como o endereço do usuário, o que dificulta a localização do paciente em caso necessário ou se surgirem possíveis erros no ato da dispensação do medicamento. Em pesquisa realizada no mesmo município deste estudo, também foi encontrado altas porcentagens de ausência do endereço do paciente, $74,75 \%{ }^{15}$.

No que se refere aos componentes de identificação do emitente, segundo a Portaria $344 / 98^{6}$ precisa ser apresentado os dados do profissional prescritor (nome e inscrição no Conselho Regional com a sigla da Unidade Federativa referente) ou dados da instituição (nome, endereço e telefone). Do total das notificações que apresentaram como emitente o profissional médico a identificação do prescritor, a assinatura e carimbo do mesmo estavam cem por cento preenchidos, sendo esses itens utilizados para a identificação do profissional em uma eventual necessidade de contato para tirar dúvidas ou obter esclarecimentos sobre a prescrição ${ }^{4}$.

Já as instituições de saúde apareceram como emitentes em 93 notificações, e em poucas delas não tinham telefone e endereço, o que dificulta a identificação da origem e contato com o prescritor para esclarecimentos de dúvidas posteriores. A presença dessas informações torna legítima a prescrição tornando-a apta para dispensação além de, facilitar a comunicação entre os profissionais envolvidos e o paciente, mitigando o risco de erros no tratamento ou desistência do recurso terapêutico por parte do usuário $^{16}$.

Foi observado no estudo uma predominância de notificações de receita prescritas por especialistas como os psiquiatras. A prescrição por esses profissionais apresenta um perfil diferenciado, uma vez que estes possuem conhecimentos aprofundados sobre as propriedades farmacológicas dos medicamentos psicoativos e os possíveis riscos associados à sua utilização $^{13}$.

Com relação ao preenchimento dos
campos relacionados ao tratamento farmacoterapêutico verifica-se o uso preferencial pela descrição do nome do medicamento de acordo com a DCB. A utilização da DCB contribui 
para a adesão ao tratamento, visto que, há uma redução no custo do medicamento para o paciente, mantendo de forma equivalente a qualidade do fármaco referência ${ }^{18}$. No caso do uso do nome comercial de medicamentos, eles podem originar inconsistências e erros à medida que estes variam dentre as indústrias produtoras e estão sujeitos a mudanças, atendendo a interesses de mercado ${ }^{21}$.

A consolidação do medicamento genérico teve como base legal a Lei no 9.787 de $1999^{22}$ com intuito de promover uma variedade de opções no mercado de medicamentos, com a finalidade de possibilitar melhorias na qualidade em âmbito nacional, redução do preço e facilidade no acesso da população aos tratamentos medicamentosos ${ }^{4}$. Dessa forma deve-se trabalhar sempre a conscientização dos profissionais de saúde para que haja prescrição conforme a legislação brasileira para atendimento no sistema único de saúde.

Em relação ao preenchimento da quantidade do medicamento, foram observados problemas como substituição da quantidade e forma farmacêutica pelo número de caixas e quantidade acima do permitido pela portaria $344 / 98^{6}$. A carência nas informações sobre os medicamentos, principalmente orientações quanto ao modo de uso, para a figura principal, que é o paciente, podem ocasionar diversos prejuízos, desde o desperdício físico do medicamento, quanto a problemas de adesão terapêutica. Além de tornar o tratamento inadequado e inefetivo, uma vez que, dificulta o alcance das metas farmacoterapêuticas estabelecidas, e por consequência, o abandono do paciente ao tratamento, trazendo o reflexo da qualidade precária no atendimento médico que interfere diretamente na dispensação ${ }^{15}$.

Além disso, ainda relacionado a quantidade, observou-se que quase a totalidade das prescrições essa variável não estava escrita por extenso ou estava preenchido em algarismos arábicos. A utilização de abreviaturas, acrônimos e símbolos simplifica a prescrição, mas pode acarretar confusão ao dispensador, paciente ou cuidador ${ }^{9}$.

Quanto à forma farmacêutica estava presente em menor parte das notificações, essa informação faz partes dos itens que colaboram para identificação do medicamento, assim como, a dose e a posologia, que estavam presentes em todas as notificações, sendo cruciais no momento da dispensação e na adesão ao tratamento. A comercialização dos medicamentos é feita em várias formas farmacêuticas, logo, a ausência desse componente pode gerar falhas na dispensação ${ }^{16}$. De forma geral, a não integralidade dos elementos que identificam o medicamento interferem negativamente no tratamento, podendo ir desde reações adversas, a recusa em aderir à terapia, além de poder gerar uma dispensação errônea ${ }^{15}$.

No que concerne à dispensação dos medicamentos psicotrópicos, a Portaria 344/98 ${ }^{6}$ estabelece o preenchimento dos itens de identificação do fornecedor (nome e endereço completo), data do atendimento e assinatura do responsável pela dispensação, além da quantidade aviada. Esses itens se referem às falhas mais observadas neste estudo, erros de dispensação, sendo que as variáveis assinatura do profissional responsável pela dispensação e carimbo não foram encontradas em nenhuma das notificações, mostrando que essa informação é negligenciada pelos profissionais farmacêuticos assim como encontrado por Pereira et $\mathrm{al}^{23}$.

Esses dados demonstram que os erros e incompletudes estão associados tanto a prescrição como a dispensação, com os profissionais responsáveis negligenciando seus papéis diante dessas atividades e da segurança do paciente. Esses profissionais são essenciais para garantir o uso racional de medicamentos e para mitigar erros de medicação. A quantidade aviada e a data de atendimento, estavam na sua totalidade preenchidas, o que assegurou que a notificação foi dispensada dentro do período de validade estabelecido em lei.

Os resultados aqui encontrados atestam a necessidade dos profissionais prescritores e dispensadores, e também, gestores estarem comprometidos com o cumprimento das leis direcionadas ao uso racional de medicamentos, para que todas as etapas do cuidado à saúde sejam realizadas corretamente e o usuário tenha um tratamento livre de erros e com segurança ${ }^{24}$. A prescrição assim como, a dispensação são ferramentas eficazes no uso racional de medicamentos. Essa última engloba muito mais do que o ato de entregar o medicamento ao paciente, mas também esclarecer e instruir quanto a utilização do medicamento, para que haja efetividade, adesão e segurança na terapia $^{11}$. 


\section{Considerações finais}

A partir dos dados obtidos foi possível avaliar as falhas nas prescrições e dispensação dos psicotrópicos das notificações de receitas B1 na unidade básica de saúde do município de JequiéBA. Verificou-se a existência de diversas falhas na completude dessas notificações de receita no que se refere a informações do paciente, do emitente, do prescritor e erros referentes às informações do medicamento e na dispensação. A presença do farmacêutico clínico, em estabelecimentos de saúde, somado ao preenchimento completo no momento da dispensação ativa, torna-se imprescindível para suprir a ausência de informações em notificações de receitas, que tem como benefício uma prevenção de erros de medicação, abuso ou uso ilícito do medicamento, garantindo ao paciente um tratamento eficaz, seguro e racional, sendo de extrema importância a conscientização do cumprimento da legislação.

\section{Referências}

1. World Health Organization. WHO. A report of the assessment of the mental health system in Brazil using the World Health Organization - Assessment Instrument for Mental Health Systems. World Health Organization. 2007;1(1):-1-51

2. Prado MAMB, Francisco PMSB, Barros MBA. Use of psychotropic medications in adults and elderly living in Campinas, São Paulo, Brazil: cross-sectional population-based study. Epidemiol. Serv. Saude. 2017;26(4):747-758.

3. Santos HS, Nestor AGS, Abreu BS, Modesto KR. A utilização dos medicamentos psicotrópicos e seus fatores associados. Rev Inic Cient Ext. 2018;1(1): 51-6.

4 .Cazarotti MLB, Lima LC, Miranda AR, Sousa EO, Bispo FCL. Psicotrópicos: Prescrições Médicas Dispensados em uma Drogaria no Município de Santa Inés - MA. Revista. Eletrônica Acerv Cien. 2019;2:e326.

5. Organización Mundial De La Salud. Plan de acción sobre salud mental 2013-2020 [Internet]. Genebra: Organización Mundial de la Salud; 2013. Disponível em: https://www.who.int/mental_health/publication s/action_plan/es/.

6. Santos AM, Felipe RL. A atuação do farmacêutico na saúde mental após a reforma psiquiátrica: uma revisão da Literatura. Residência Multiprofissional em Área Profissional da saúde UFU. 2018;1:18-5.

7. Brasil. Secretaria De Vigilância Sanitária Do Ministério Da Saúde. Portaria n 344, de 12 de maio de 1998. Aprova o Regulamento Técnico sobre substância e medicamentos sujeitos a controle especial. Disponível em: https://bvsms.saude.gov.br/bvs/saudelegis/svs/1 998/prt0344_12_05_1998_rep.html.

8. Arruda EL, Hevilem LMN, Morais AKP. Avaliação das informações contidas em receitas e notificações de receitas atendidas na farmácia do CAPS II Araguaína-TO. Revista Científica do ITPAC, Araguaína. 2012;5(2):10-2.

9. Alves CDS, Santos RG, Lemos GS. Análise da Completude das Prescrições Médicas de Antimicrobianos dispensadas em Farmácia Comunitária de Jequié, Bahia. Revista Baiana de Saúde Pública. 2017;41(1): 29-44.

10. Brasil. Presidência Da República. Lei no 13.021, de 8 de agosto de 2014. Dispõe sobre o exercício e a fiscalização das atividades farmacêuticas. Disponível em: http://www.planalto.gov.br/ccivil_03/_Ato20112014/2014/Lei/L13021.htm.

11. Moraes GS, Almeida PHRF, Lemos LB, Lemos GS. Anabolizantes: erros de prescrição e dispensação. J Manag Prim Health Care. 2020;1(12):16-1.

12. Azevedo LS, Pereira LJ, Zangerônimo MG, Sousa RV, Murgas LDS, Marques LS et al. Avaliação da adequação legal de receitas e notificações de receita de medicamentos sujeitos a controle especial dos setores públicos e privados. Revista de Ciências. Farmacêuticas Básica Aplicada. 2011;32(3):401-417.

13. Ferrari CKB, Brito LF, Oliveira CC, Moraes EV, Toledo OR, David FL. Falhas na Prescrição e Dispensação de Medicamentos Psicotrópicos: Um problema de Saúde Pública. Rev de Ciências Farmacêuticas Básic Aplic. 2013;34(1):109-116.

14. Mota IVR, Nascimento LES, Cardoso RA, Lemos LB, Lemos GS. Medicamentos sob controle especial: uma análise dos erros de medicação e indicadores de prescrição. Rev Eletrônica Farm. 2016;13(1):45-54

15. Pinheiro MTRS, Souza SSS, Almeida PHRF, Lemos LB, Lemos GS. Falhas e erros em notificações de receitas de metilfenidato dispensadas em uma farmácia comunitária. InterScientia. 2016;4(2):58-66.

16. Souza SSS, Pinheiro MTRS, Almeida PHRF, Lemos LB, Lemos GS. Sibutramina: falhas e incompletude de documentos na prescrição e dispensação. Rev. Aten. Saúde. 2017;15(51): 2333.

17. Cardoso RS, Mota IVR, Lemos LB, Almeida PHRF, Rosa MB, Lemos GS. Erros em 
receitas de controle especial e notificações de receita prescritas por profissionais não médicos. Rev. Aten. Saúde. 2018;16(55):42-1.

18. Santana LSOS, Carvalho TS, Martins IVO, Costa SJ, Sousa LFL, Sousa Jr G. Análise dos aspectos clínicos e deontológicos de prescrições provenientes de uma farmácia hospitalar de Teresina -PI. Rev de Casos e Consultoria.2020;11(1):1-11

19. World Health Organization. WHO. Medication without harm: WHO's third global patient safety challenge. Geneva: WHO, [2017]. Disponível em: $<$ http://www.who.int/patientsafety/medicationsafety/en/>

20. Cunha MMC, Miceli BC. Análise de prescrições médicas de psicotrópicos em uma drogaria no município de Sete Lagoas. Rev Bras de Ciên da Vida. 2017;5(5):17-1

21. Rocha, APS. Perfil da notificação de psicotrópico B1 dispensada em uma unidade básica de saúde do município de Lagarto-SE. 2019.

22. Brasil. Ministério da Saúde. Lei n.9.787, de 10 de fevereiro de 1999. Estabelece o medicamento genérico, dispõe sobre a utilização de nomes genéricos em produtos farmacêuticos e dá outras providências. Disponível em: https://www.planalto.gov.br/ccivil_03/Leis/L978 7.htm.

23. Pereira IOE, Lemos LB, Almeida PHRF, Lemos GS. Erros de Prescrição e dispensação de antimicrobianos em uma farmácia comunitária. Saúde.com. 2020 ;15(4): 1690-1699.

24. Gonçalves MF, Rosas BOA, Pacheco TM, Ferreira RG, Lobo LG. Prescrição médica e o uso irracional de medicamentos: uma revisão bibliográfica. Rev Bioética Cremego. 2020;1(1):55-60.

\section{Endereço para Correspondência}

Gisele da Silveira Lemos

E-mail: giselesilveiralemos@gmail.com

Recebido em 03/09/2021

Aprovado em 23/12/2021

Publicado em 30/12/2021 